\title{
Computational prediction and experimental validation of a novel miRNA in Suaeda maritima, a halophyte
}

\author{
S.A. Gharat and B.P. Shaw \\ Environmental Biotechnology Laboratory, Institute of Life Sciences, Bhubaneswar, \\ Odisha, India \\ Corresponding author: S.A. Gharat \\ E-mail: sachingharat113@gmail.com \\ Genet. Mol. Res. 15 (1): gmr.15017527 \\ Received August 25, 2015 \\ Accepted November 27, 2015 \\ Published January 22, 2016 \\ DOI http://dx.doi.org/10.4238/gmr.15017527
}

ABSTRACT. The lack of available transcriptome data for plants of no economic or agronomic importance limits the identification of miRNAs in many species. Considering the possible similarity of the transcriptome between related species, the present study used expressed sequence tags (ESTs) of Suaeda salsa and Suaeda glauca to identify conserved miRNAs, which were validated in a halophyte, Suaeda maritima, with the aim of identifying salt-responsive miRNAs from naturally salt-tolerant plants, information on which is limited. In this study, computational analysis predicted three miRNA sequences by mapping non-redundant miRNA sequences from miRBase 16.0 on 1534 ESTs of $S$. salsa and $S$. glauca. The expression of one could be validated in S. maritima, and was named sma-miR1867. This miRNA was downregulated in response to $\mathrm{NaCl}$ treatment. It was predicted to target ferredoxin-thioredoxin reductase (FTR), cell division control protein 6 (CDC6), and ubiquitinprotein ligase (UPL) in S. salsa and/or S. glauca. However, only UPL could be amplified in S. maritima, and RT-qPCR showed that it was upregulated in response to $\mathrm{NaCl}$ treatment. These results indicate that, in halophytes, FTR and CDC6 may promote carbon metabolism and cell division, respectively, in the presence of salt, while UPL may regulate the abundance of proteins that are important for salt tolerance in halophytes. 
Thus, sma-miR1867 could be an essential component of salt resistance in halophytes.

Key words: NaCl salinity; Suaeda salsa; Suaeda glauca; sma-miR1867; Abiotic stress; Halophyte

\section{INTRODUCTION}

Biochemical and physiological processes in plants and animals are strictly regulated by various mechanisms at different levels. In this regard, micro RNAs (miRNAs) function as repressors of gene expression through post-transcriptional regulation in all known animal and plant genomes. In plants, miRNAs have been reported to regulate plant growth and development, including leaf morphogenesis, lateral root formation, growth stage transition from juvenile to adult and vegetative to flowering, floral organ development, and reproduction (Mallory and Vaucheret, 2006). Furthermore, a regulatory role of miRNAs in biotic and abiotic responses in plants has also been indicated (Sunkar and Zhu, 2004; Ding et al., 2009; Pandey et al., 2013).

Among abiotic factors, high salinity is a severe and increasing constraint on global crop production, as most of the major crops are highly susceptible to salinity; therefore, there is an urgent need to address this problem. However, our understanding of the mechanism of salt tolerance is far from clear, and discovery of salt-responsiveness miRNAs has added complexity to this already complex mechanism (Sunkar and Zhu, 2004;Ding et al., 2009; De Paola et al., 2012). Sunkar and Zhu (2004) showed, for the first time, that upregulation of miR393, miR402, miR397b, and miR319c occurred in response to at least one of the following stresses; drought, cold, and salt. However, with regard to salt-responsiveness, research has mostly been carried out on test species that are either sensitive or naturally not tolerant to salt (Ding et al., 2009; De Paola et al., 2012), with the exclusion of studies by Khraiwesh et al. (2013) and Feng et al. (2015).

Identification of miRNAs in a species requires prior information on their sequence from other species or information on the small-RNA (sRNA) sequence from the same species obtained through cloning and sequencing. Second, knowledge of the genome or transcript sequences of the species permits the unique secondary structure of the pri-miRNA to be established computationally (Jones-Rhoades and Bartel, 2004; Zhang et al., 2005). A lack of genome/transcriptome/expressed sequence tag (EST) data restricts the identification of miRNAs in a species despite the availability of miRNA sequences from many sources. This is particularly true for plants that are of no economic or agronomic importance, such as halophytes, which have been paid little attention in terms of their genomes. However, it has been increasingly realized that many miRNAs are conserved evolutionarily in the plant kingdom and that conservation occurs from lower plants, including mosses and ferns, to higher flowering plants, and hence it is very likely that miRNAs identified through a computational approach considering ESTs of a plant species will be present in a closely related species for which an EST database is not available. Computational approaches have been very effective, especially in plants in which miRNA and target mRNA have often nearly perfect complementarity (Jones-Rhoades and Bartel, 2004; Zhang et al., 2005). Therefore, the present study takes advantage of the availability of ESTs for Suaeda salsa and Suaeda glauca in order to identify miRNAs and their precursors in a closely related species, Suaeda maritima, which is a halophyte, considering the availability of scant information on salt-responsive miRNAs from a naturally salt-tolerant plant. 


\section{MATERIAL AND METHODS}

\section{Prediction of potential miRNAs in Suaeda ESTs}

To identify potential salt-responsive miRNAs in a halophyte, all plant miRNAs were downloaded from the miRNA Registry Database (miRBase 16.0, http://www.sanger.ac.uk/). The repetitive sequences were removed and the remaining non-redundant sequences were used as reference miRNAs. A total of 1534 EST sequences of S. salsa and S. glauca were retrieved from the NCBI database (http://www.ncbi.nlm.nih.gov/). All non-redundant plant miRNAs were subjected to a BLASTn search of the retrieved ESTs sequences of the Suaeda species. Considering that miRBase is not rich in miRNAs from mangrove and/or halophytes, the ESTs sequences with 0-5 mismatches against the plant miRNAs were selected, instead of 0-4 mismatches, which is preferred (Meyers et al., 2008). These ESTs were subjected to a BLASTx search to remove the ESTs coding for known proteins. The remaining ESTs were used to predict the hairpin structure by the mfold software with default parameters (http://mfold.rna.albany.edu/). The length of the precursor was reduced until the minimal folding free energy index (MFEI) was maximal. Furthermore, it was also ensured that the precursors were at least $60 \mathrm{nt}$ long, the secondary structure displayed an appropriate stem loop hairpin, the miRNA sequence occupied only one arm of the hairpin, the maximum number of bases were present in the stem of the stem-loop hairpin structure, and the mature miRNA sequence and its opposing miRNA strand $\left(\mathrm{miRNA}^{*}\right)$ did not contain more than six nt mismatches (Zhang et al., 2005). The MFEI value was calculated as per the equation: MFEI = [(MFE/length of the precursor miRNA sequence) X 100] / (G + C)\% (Zhang et al., 2006a).

\section{Test plant and $\mathrm{NaCl}$ application}

Seeds of S. maritima L. were collected from adult plants growing along the mangrove coastal belt in Bhadrak $\left(21.13^{\circ} \mathrm{N}, 86.76^{\circ} \mathrm{E}\right)$, Odisha, India. The seeds were spread on autoclaved soil mixed with sand in plastic pots with holes in the bottom and were watered every day, alternating between 1/10th Hoagland's solution and Milli-Q water. The seedlings were grown in a greenhouse maintained at $24^{\circ} \pm 3^{\circ} \mathrm{C}$ and $70-75 \%$ relative humidity under a natural day/night cycle with provision

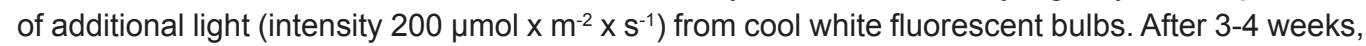
the seedlings were approximately $2 \mathrm{~cm}$ in height. At this stage, groups of three seedlings were transferred to separate plastic pots and were set to acclimatize and grow for $\sim 3$ months in the greenhouse. The individual pots were watered every day, alternating between 1/10th Hoagland's solution and Milli-Q water except on the penultimate day when $\mathrm{NaCl}$ was applied. Initially $500 \mathrm{~mL}$ $0.5 \% \mathrm{NaCl}$ prepared in $1 / 10$ th strength Hoagland's solution was poured into the individual pots early in the morning. The control pots received only 1/10th Hoagland's solution. After $30 \mathrm{~min}, 100$ $\mathrm{mL} 2.0 \% \mathrm{NaCl}$ prepared in 1/10th strength Hoagland's solution was poured into the treated pots at 30 min intervals. After 9-h exposure, the aerial portions of the test plants were excised, preserved in liquid $\mathrm{N}_{2}$, and stored at $-80^{\circ} \mathrm{C}$ until analysis.

\section{Expression study of the predicted miRNAs}

Total RNA was isolated from preserved shoot samples of the control and $2.0 \% \mathrm{NaCl}-$ treated $(9$ h) S. maritima plants using a miRNeasy mini kit (Qiagen) following the manufacturer 
protocol. For northern blot analysis, $10 \mu \mathrm{g}$ total RNA was resolved on a $15 \%$ urea-polyacrylamide gel electrophoresis and transferred on to nylon membrane using Trans-Blot ${ }^{\circledR}$ SD Semi-Dry Electrophoretic Transfer Cell (Bio-Rad). The blot was air dried and UV-crossed linked at 150 mJ using a UV cross linker (HoeferTM UVC 500 Cross linker). The sense and anti-sense DNA oligonucleotide probes were end-labeled individually with $\left[{ }^{32} \mathrm{P}\right] \gamma$-dATP using T4 Polynucleotide Kinase (Fermentas) according to the manufacturer instructions. Individual membrane blots were pre-hybridized for $1 \mathrm{~h}$ with hybridization buffer (Sigma). The radiolabelled probe was added individually to each membrane and hybridization continued for $16 \mathrm{~h}$ at $37^{\circ} \mathrm{C}$. After hybridization, the membrane was washed with 2 X SSC (saline sodium citrate), $0.1 \%$ SDS (sodium dodecyl sulfate) at $32^{\circ} \mathrm{C}$ for $15 \mathrm{~min}$ and finally with $1 \mathrm{X} \mathrm{SSC}$ and $0.1 \%$ SDS for $15 \mathrm{~min}$ at $37^{\circ} \mathrm{C}$. The membrane was air dried and then exposed to X-ray film. When needed, membranes were stripped, re-exposed to X-ray film to ensure complete signal removal, and reused for a second hybridization. A DNA oligonucleotide complementary to U6 small nuclear RNA (snRNA) was used as a probe to detect the U6 snRNA as a reference control for normalization.

\section{Expression study of miRNA precursor by RT-PCR}

RT-PCR was carried out to determine changes in the expression of the sma-miR1867 precursor in response to $\mathrm{NaCl}$ application. Total RNA from the preserved control and $2.0 \% \mathrm{NaCl}$ treated (9 h) S. maritima shoot samples was isolated as described above to prepare cDNA using a SuperScript ${ }^{\circledR}$ III First-Strand Synthesis kit from Invitrogen following the manufacturer protocol. Briefly, $1 \mu \mathrm{g}$ DNase treated total RNA, $1 \mu \mathrm{L}$ dNTP mix (10 mM each), and $1 \mu \mathrm{L}$ random hexamer were added to a 2-mL PCR tube and incubated at $65^{\circ} \mathrm{C}$ for $5 \mathrm{~min}$ followed by incubation on ice for $\sim 5$ min. In the same tube, $4 \mu \mathrm{L} 5 \mathrm{X}$ First Strand Buffer, $1 \mu \mathrm{L}$ 0.1M DTT, $1 \mu \mathrm{L}$ RNaseOUT (40 U/1 $\mu \mathrm{L})$, and $1 \mu \mathrm{L}(200 \mathrm{U})$ SuperScript III reverse transcriptase was added to a final volume of $20 \mu \mathrm{L}$. Next, the contents of the PCR tube were incubated at $50^{\circ} \mathrm{C}$ for $1 \mathrm{~h}$ followed by $70^{\circ} \mathrm{C}$ for $15 \mathrm{~min}$, and finally at $4^{\circ} \mathrm{C}$ for $5 \mathrm{~min}$. The prepared $1 \mathrm{st}$ strand cDNA was used as a template for amplification of the sma-miR1867 precursor using primers flanking the miRNA1867 region. PCR amplification was carried out on a DNA engine (Bio-Rad) thermo cycler taking cDNA synthesized as a template and using GoTaq ${ }^{\circledR}$ DNA polymerase (Promega) and PCR nucleotide mix (Promega) following the protocol detailed in the manual. The PCR program was set as $94^{\circ} \mathrm{C}$ for 3 min followed by 30 cycles of $94^{\circ} \mathrm{C}$ for $30 \mathrm{~s}, 59^{\circ} \mathrm{C}$ for $30 \mathrm{~s}, 72^{\circ} \mathrm{C}$ for $30 \mathrm{~s}$, and finally, $72^{\circ} \mathrm{C}$ for $5 \mathrm{~min}$. The amplified products $(7 \mu \mathrm{L})$ were resolved on $2.0 \%$ agarose gel and visualized by ethidium bromide staining and UV illumination. Actin served as a loading control.

\section{Cloning and sequencing of precursor amplified by RT-PCR}

The precursor amplified by RT-PCR was cloned in pGEMT-Easy vector and transformed into JM109 competent cells. The transformed bacteria were plated on LB/agar plates containing ampicillin. For blue-white colony screening, $100 \mu \mathrm{L} 100$ mM IPTG (isopropyl $\beta$-D-1-thiogalactopyranoside) and $20 \mu \mathrm{L} 50 \mu \mathrm{g} / \mathrm{mL}$ X-Gal were spread on each plate. Several white colonies were isolated and the insert was amplified by colony PCR. The reaction was performed with an initial denaturation at $94^{\circ} \mathrm{C}$ for 5 min, followed by 35 cycles of $94^{\circ} \mathrm{C}$ for $30 \mathrm{~s}, 60^{\circ} \mathrm{C}$ for $30 \mathrm{~s}$, and $72^{\circ} \mathrm{C}$ for $30 \mathrm{~s}$, and a final extension at $72^{\circ} \mathrm{C}$ for $5 \mathrm{~min}$. The amplicons were sequenced using SP6 and T7 vector specific primers on an Applied Biosystem DNA sequencer (Genetic analyzer 3500). 


\section{Expression analysis of miRNA target by RT-qPCR}

Total RNA was extracted and first-strand CDNA was synthesized from the control and $\mathrm{NaCl}$ treated S. maritima samples as described. A QuantiTect SYBR Green PCR Kit (Qiagen) was used for the RT-qPCR on a LightCycler ${ }^{\circledR} 480$ Real-Time PCR System (Roche). The reactions were individually run in $20-\mu \mathrm{L}$ volumes in 96 -well plates as detailed in the manual. Actin expression in each cDNA preparation served a reference. The relative expression/abundance of individual target genes in $\mathrm{NaCl}$-treated samples compared with the control sample was calculated using the $2^{-\Delta \Delta \mathrm{Ct}}$ method considering actin templates in the sample as the reference level.

\section{sma-miR1867 target prediction}

Target prediction was carried out using psRNATarget tools (http://plantgrn.noble.org/ psRNATarget/) considering the EST sequences of the halophytes $S$. salsa and $S$. glauca that were available at the NCBI database with default parameters. Considering the availability of only 1534 ESTs from these two species in the database, the target prediction was performed with less stringency $(E$ values $\leq 4)$ than usually followed $(E \leq 3)$. The predicted target transcript sequences were BLASTx searched through the NCBI site for annotation of their probable function.

\section{RESULTS AND DISCUSSION}

The identification and characterization of miRNAs are widely pursued research fields for multiple reasons. First, the number of miRNAs identified is still relatively low; second, the number of species covered so far is low, particularly in plants, considering their wide taxonomical and ecological diversity; and third, this subject can be applied to all fields of research in modern biology. Furthermore, the discovery of miRNAs has added another dimension to molecular biology research concerned with understanding the regulatory aspects of molecular events that maintain life under extreme environmental conditions. Being sessile, plants have evolved to adapt to harsh environmental conditions, and recently, a number of miRNAs have been shown to be sensitive to abiotic stresses (Sunkar and Zhu, 2004) indicating their importance in the adaptive process (Jones-Rhoades and Bartel, 2004; Sunkar and Zhu, 2004). However, all such miRNAs have been identified by deep sequencing of sRNA followed by mapping of the sequences with the ESTs of the species under investigation (Jones-Rhoades and Bartel, 2004; Sunkar and Zhu, 2004). Recently, Pandey et al., (2013) used a computational approach to identify drought stress-responsive miRNAs in wheat using abiotic stressed cDNA libraries for this plant. However, the results of the current study show that ESTs of related species can be used to successfully identify miRNAs using a computational approach.

Three potential miRNAs were predicted using 1534 ESTs of S. salsa and S. glauca and non-redundant miRNA sequences from miRBase (Table 1) showing mismatches at a maximum five nucleotide positions (Figure 1). The predicted conserved miRNA sequences belonged to the miR529, miR1867, and miR2657 families. The negative MFE of precursor miRNAs varied significantly within the range -28.20 to $-40.10 \mathrm{kcal} / \mathrm{mol}$, the length of the precursors was greater than the desired length of $60 \mathrm{nt}$, the miRNAs occupied only one arm of the concerned secondary structure, and there were no more than six mismatches between the miRNA/miRNA* duplex (Table 1, Figure 2), suggesting that the precursors could be diced to form mature miRNAs (Zhang 
et al., 2005). The $A+U$ contents of the precursors varied from 57 to $71.63 \%$ (Table 2), which is consistent with previous reports (Zhang et al., 2006b). The MFEl values of the miR529, miR2657, and miR1867 precursors were $0.46,0.62$, and 0.72 , respectively (Table 1 ). Among these, the MFEI of the miR1867 precursor was higher than that of other non-coding RNAs indicating that this is the most likely candidate miRNA precursor (Zhang et al., 2006a).

Table 1. Chemical features of predicted miRNAs and their precursors.

\begin{tabular}{|c|c|c|c|c|c|c|c|c|}
\hline SI. No. & miRNA predicted & Gene ID & Loc & LM (nt) & LP (nt) & $\mathrm{G}+\mathrm{C}(\%)$ & $\operatorname{MFE}(-\Delta G)$ & MFEI \\
\hline 1 & miR529 & gi|9057119 & $5^{\prime}$ & 21 & 213 & 28.63 & 28.20 & 0.46 \\
\hline 2 & miR1867 & gi|9057160 & $3^{\prime}$ & 23 & 128 & 42.96 & 40.10 & 0.72 \\
\hline 3 & $\operatorname{miR} 2657$ & gi|314999170 & $3^{\prime}$ & 22 & 208 & 28.36 & 36.80 & 0.62 \\
\hline
\end{tabular}

Loc: location of mature miRNA in the stem-loop, LM: length of the mature miRNA, LP: length of the precursor, MFE: minimal folding free energy, MFEl: minimal folding free energy index.

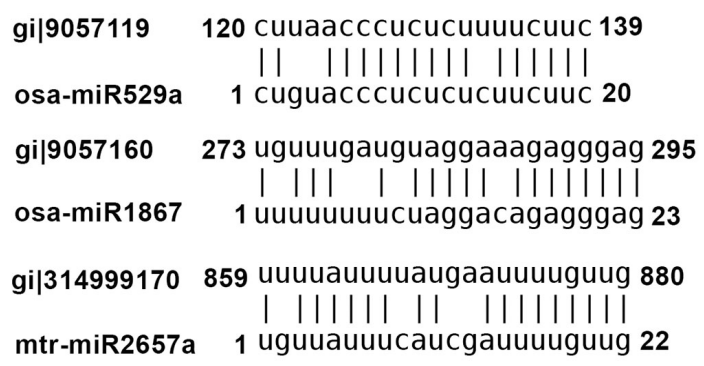

Figure 1. Nucleotide base pairing of the miRNAs predicted with those available in miRBase.

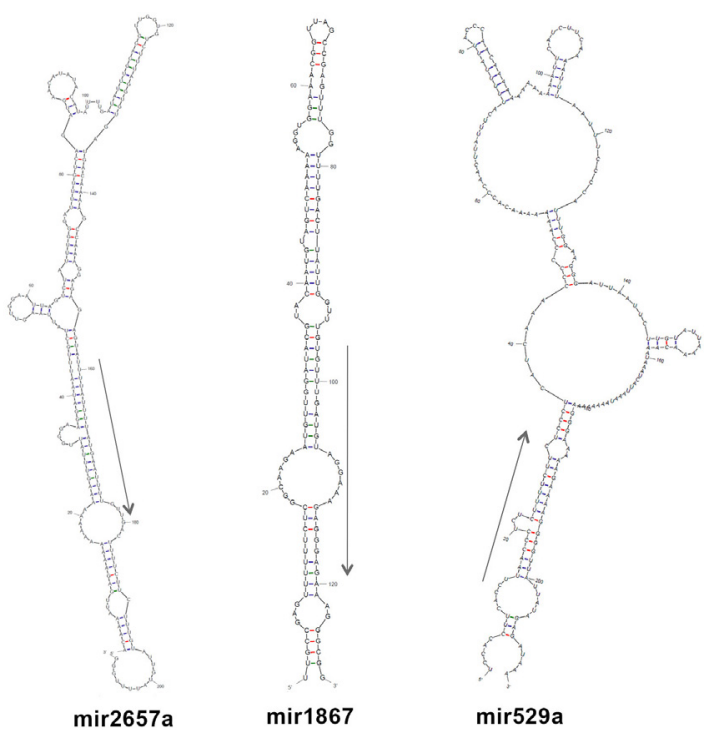

Figure 2. Stem-loop secondary structure of predicted miRNA precursors. The arrow in each secondary structure indicates the miRNA sequence. 
Table 2. Nucleotide composition of the predicted miRNA precursors.

\begin{tabular}{lcccccccc}
\hline Sr. No. & miRNAs predicted & A $\%$ & C $\%$ & G\% & U\% & A/U ratio & G/C ratio & A+U (\%) \\
\hline 1 & mir529 & 42.25 & 21.12 & 7.51 & 29.10 & 1.45 & 0.35 & 71.36 \\
2 & mir1867 & 25.78 & 10.15 & 32.81 & 31.25 & 0.82 & 3.23 & 57.00 \\
3 & mir2657 & 31.73 & 6.25 & 22.11 & 39.90 & 0.79 & 3.53 & 71.63 \\
\hline
\end{tabular}

Of the three antisense oligo-probes, only that for miR1867 generated a signal in northern blot hybridization (Figure 3-I), indicating that it is expressed in the plant (S. maritima) fulfilling the miRNA annotation criteria (Ambros et al., 2003). Its expression was detected in both control and $\mathrm{NaCl}$-treated plants and was named sma-miR1867 as per the nomenclature criteria set forth by Ambros et al. (2003). When predicted miRNAs were not detected by northern blot hybridization in control or $\mathrm{NaCl}$-treated plants, they were considered to be absent in S. maritima. Northern blot hybridization performed with DNA oligonucleotides identical to sma-miR1867 did not produce any signals, confirming that miRNA sma-miR1867 is expressed in S. maritima (Figure 3-II); since siRNAs are derived from sense and antisense RNA duplexes, a hybridization signal with the identical oligonucleotide would be expected if sma-miR1867 was a siRNA (Jian et al., 2010). Densitometric analysis of the sma-miR1867 northern blot hybridization signal revealed that the expression of this miRNA was downregulated in $\mathrm{NaCl}$-treated plants (Figure 3-III) suggesting its importance in the salt tolerance process.
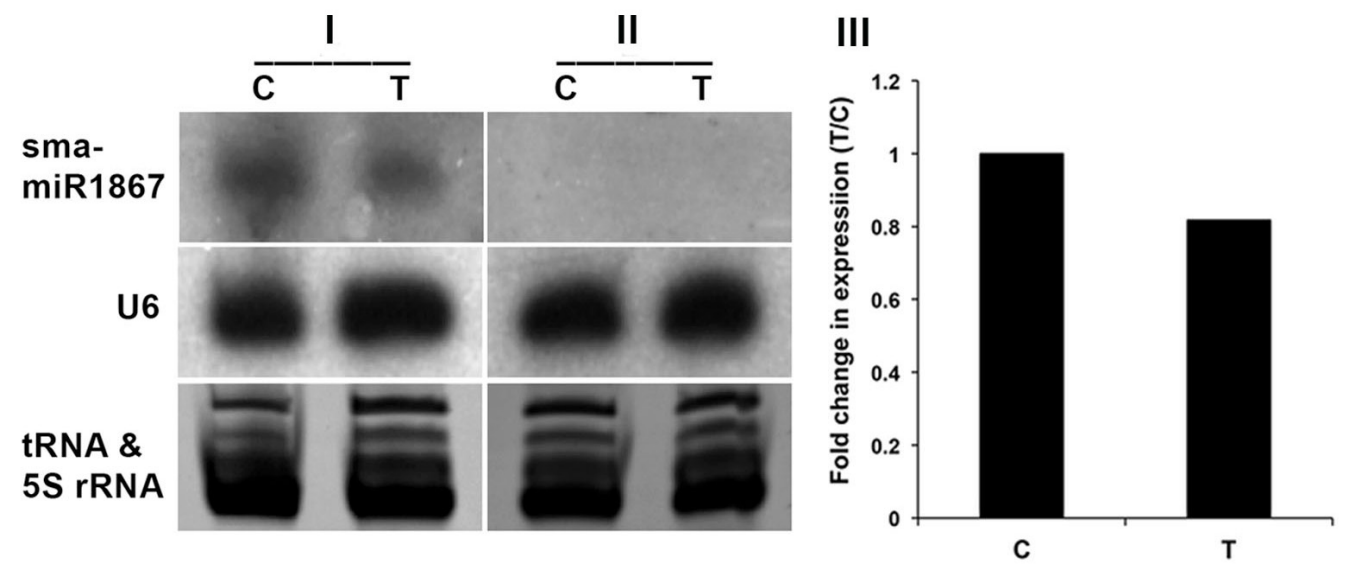

Figure 3. Northern blot hybridization confirming the expression of sma-miR1867 in Suaeda maritima. The RNA blots were probed either with a $\left.{ }^{32} \mathrm{P}\right]$ end-labeled oligo complimentary to sma-miR1867 (I), or with a $\left[{ }^{32} \mathrm{P}\right]$ end-labeled oligo identical to sma-miR1867 (II). In each blot, lanes C and T represent RNA isolated from control and 2.0\% NaCl-treated plants, respectively. Only blot-I showed a hybridization signal of $\sim 20-24$ nt in size suggesting that the predicted miRNA sequence, sma-miR1867, is a miRNA. Both blots were probed with a radio-labeled oligonucleotide complementary to U6 snRNA for use as a loading control. tRNA/5S rRNA bands were also visualized by ethidium bromide staining of polyacrylamide gels before blotting. (III) Densitometric representation of the hybridization signal in blot-I showing downregulation of the miRNA upon $\mathrm{NaCl}$-treatment. 
RT-PCR analysis using S. maritima cDNA and the primer pairs designed to amplify the sma-miR1867 precursor revealed an amplified product of the desired length (Figure 4-I). Sequencing of the cloned RT-PCR product of the sma-miR1867 precursor and alignment of the sequence to the EST of $S$. salsa revealed complete homology, with the two sequences differing at only two base positions (Figure 4-II). Amplification of this precursor from S. maritima confirmed that sma-miR1867 is expressed in S. maritima and also suggests that miR1867 is well conserved in plants. Deep sequencing of rice spikelets has also revealed the presence of miR1867 (Jian et al., 2010). Furthermore, expression of miR1867 has also been confirmed in Triticum dicoccoides (Kantar et al., 2011) and Brachypodium distachyon (Budak and Akpinar, 2011) by qRT-PCR (stemloop PCR) and microarray analysis, respectively, providing further evidence of its conservation in plant species across taxonomic groups.

The expression of sma-miR1867 was downregulated following treatment with $2.0 \% \mathrm{NaCl}$, which is the concentration of $\mathrm{NaCl}$ present in seawater (Figure 3-III). RT-qPCR also revealed a decrease in the level of sma-miR1867 precursor expression following $\mathrm{NaCl}$ treatment (Figure 5), confirming that the expression of this miRNA is regulated by salt stress. In addition, miR1867 has also been reported to be responsive to dehydration stress (Budak and Akpinar, 2011; Kantar et al., 2011). However, in contrast to salt stress, dehydration stress upregulated the expression of miR1867. Moreover, miR1867 was also upregulated in rice spikelets towards the late stage of grain filling, i.e., when the moisture content of the grains started to decrease (Peng et al., 2014), simulating dehydration stress. Hence, miR1867 seems to respond differently depending on the abiotic stress encountered to fulfill the requirement for altered metabolism under given conditions.

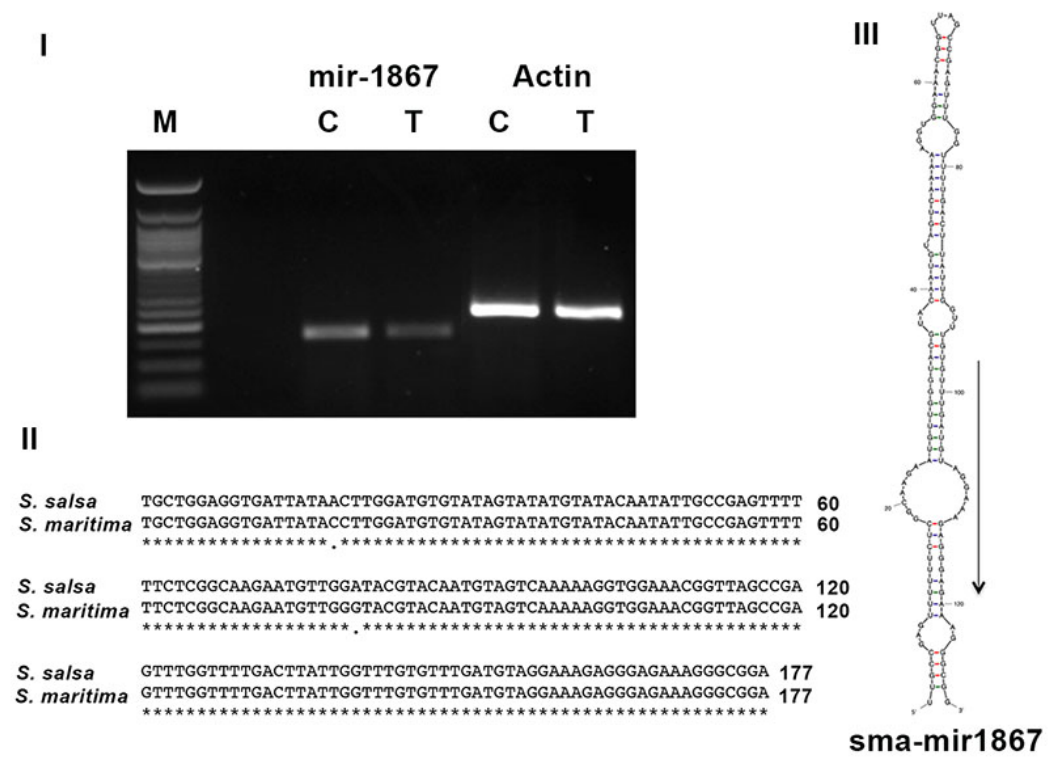

Figure 4. Expression analysis of the sma-miR1867 precursor by RT-PCR in Suaeda maritima and its secondary structure. (I). 1st strand cDNA was synthesized by reverse transcription of the total RNA and used in PCR. PCR was carried out for 30 cycles and the amplified products $(7 \mu \mathrm{L}$ each) were resolved on $2 \%$ agarose gel and visualized by ethidium bromide staining and UV illumination. Actin was used as a loading control. M: 50-bp DNA ladder, C: RNA from control plant, T: RNA from the plant treated with $2.0 \% \mathrm{NaCl}$ for $9 \mathrm{~h}$. (II). The amplified sma-mir1867 fragment was sequenced and aligned with that of the Suaeda salsa EST. (III). Secondary structure of sma-mir1867. 


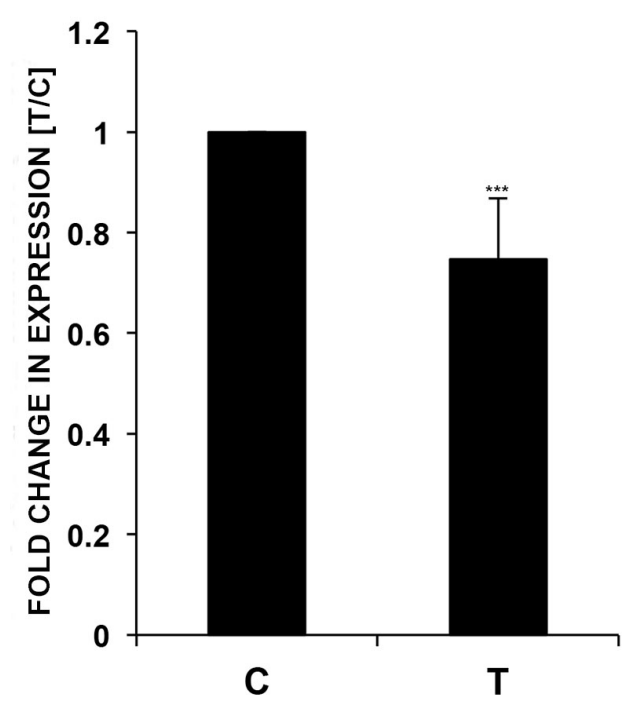

Figure 5. RT-qPCR of the sma-miR1867 precursor in Suaeda maritima showing its downregulation in response to $\mathrm{NaCl}$ application. Data are reported as means $\pm \mathrm{SD}$ of three replicate studies. ${ }^{* *}$ Significant difference at $\mathrm{P} \leq 0.001$.

Three targets of known function were predicted for sma-miR1867 considering the ESTs of the halophytes (Table 3). These were the ferredoxin-thioredoxin reductase catalytic chain, the cell division control protein CDC6 and ubiquitin protein ligase (Table 3). Ferredoxin-thioredoxin reductase catalyzes the electron transfer between ferredoxin, the first soluble electron acceptor of the photosynthetic electron transfer chain, and thioredoxin, a ubiquitous protein, which is efficient at exchanging thiol-disulfide with various protein disulfides because of its active site dithiol. Reduced thioredoxin can reduce disulfides of several target enzymes, most of which are weakly active or inactive in the oxidized form. Regulatory enzymes of a number of fundamental chloroplast processes have been reported to be linked to thioredoxin, including glucose 6-phosphate dehydrogenase of the oxidative pentose phosphate cycle, ATP synthase of ATP synthesis, NADP-malate dehydrogenase of C4 photosynthesis, fructose 1, 6-bisphosphatase, sedoheptulose 1,7-bisphosphatase, phosphoribulokinase, NADP-glyceraldehyde 3-phosphate dehydrogenase, and Rubisco of the Calvin-Benson cycle (Buchanan et al., 2002). Thus, $\mathrm{NaCl}$ application seems to stimulate photosynthetic responses in halophytes by enhancing the formation of reduced thioredoxin through downregulation of sma-miR1867 expression. Similarly, cell division also seems to be promoted in halophytes following $\mathrm{NaCl}$ application, as sma-miR1867 also targets the cell division control protein CDC6, which is essential for the initiation of DNA replication (Masuda et al., 2004).

Table 3. Characterization of sma-miR1867 expression in Suaeda maritima.

\begin{tabular}{|c|c|c|c|}
\hline Target accesstion No. & Target description & Inhibition type & miRNA/mRNA pair \\
\hline >gi|314999457 & Ferredoxin-thioredoxin reductase catalytic chain & Cleavage & .:..::::::.::::::::.:.: \\
\hline$>$ >gi|9057103 & Cell division control protein CDC6 & Translation & ::::::::::::::::::: \\
\hline$>$ >gi|9057157 & Ubiquitin-protein ligase & Cleavage & ::::::::::::::: \\
\hline
\end{tabular}


Among the three targets identified, it was possible to amplify the target gene ubiquitinprotein ligase from S. maritima by designing primers based on the EST of S. salsa. Sequencing of the amplified fragment revealed nearly perfect identity with S. salsa ESTs (Figure 6-I). Furthermore, RT-qPCR revealed significant upregulation of the target transcript (Figure 6-II) providing indirect evidence that the ubiquitin-protein ligase gene product is the target of sma-miR1867. Ubiquitinprotein ligase serves as a unit of the ubiquitin/26S proteasome system, which is involved in polyubiquitination-mediated degradation of soluble proteins and influences their activity and abundance and thus affecting a variety of cellular functions. Suppression of the miRNA (seu-miR396h/l) that targets ubiquitin-specific protease, which is involved in protein degradation, has also been reported in the euhalophyte $S$. europaea during long-term salt treatment (Feng et al., 2015). The biological role of the ubiquitin/26S proteasome system has been investigated in relation to abiotic stress tolerance in plants, which can be positively or negatively regulated (Stone, 2014). Therefore, regulation of the activity and abundance of both membrane and cytosolic proteins appears to be important for enabling plants to grow in saline environments.

S. maritima TGAAGGGGAGATTGAACGTCCAGTTTCAAGGTGAAGAAGGTATTGATGCTGGTGGCCTCA 60

S. salsa TGAAGGGGAGATTGAACGTCCAGTTTCAAGGTGAAGAAGGTATTGATGCTGGTGGCCTCA 60

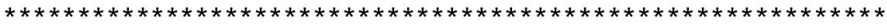

s. maritima CTAGAGAGTGGTACCAGCTATTGTCGAGGGTCATCTTTGATAAGGGAGCTCTACTCTTTA 120

s. salsa CTAGAGAGTGGTACCAGCTATTGTCGAGGGTCATCTTTGATAAGGGAGCTCTACTCTTTA 120 $* * * * * * * * * * * * * * * * * * * * * * * * * * * * * * * * * * * * * * * * * * * * * * * * * * * * * * * * * * * *$

s. maritima CGACAGTTGGAAATAATGCGACCTTCCAGCCAAACCCTAATTCTGTCTATCAGACTGAAC 180

s. salsa CGACAGTTGGGAATAATGCGACCTTCCAGCCAAACCCTAATTCTGTCTATCAGACTGAAC 180 $* * * * * * * * * * * * * * * * * * * * * * * * * * * * * * * * * * * * * * * * * * * * * * * * * * * * * * * * * * *$

s. maritima ATCTTTCTTACTTCAAATTTGTTGGTCGTGTGGTTGCAAAGGCATTATTCGATGGGCAGC 240

s. salsa ATCTTTCTTACTTCAAATTTGTTGGTCGTGTGGTTGCAAAGGCCTTATTCGATGGGCAGC 240

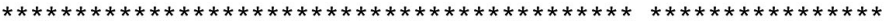

S. maritima TTTTGGATG 249

S. salsa TTTTGGATG 249

II $* * * * * * * * *$

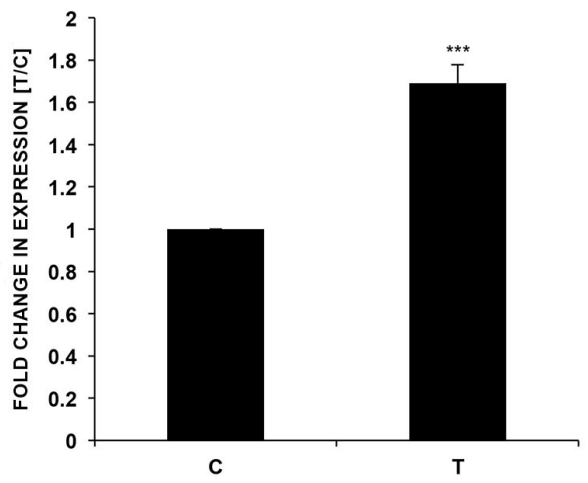

Figure 6. PCR amplification and RT-qPCR of the sma-miR1867 target ubiquitin-protein ligase. (I). RT-PCR amplified target fragment in Suaeda maritima was sequenced and aligned with the EST of S. salsa using Clustal Omega. (II). RT-qPCR of ubiquitin-protein ligase in $S$. maritima showing its upregulation in response to $\mathrm{NaCl}$ application. Data are reported as means \pm SD of 3 replicate studies. ${ }^{* *}$ Significant difference at $\mathrm{P} \leq 0.001$. 
In conclusion, this study predicted three miRNAs from 1534 ESTs of $S$. salsa and S. glauca present in the NCBI database through a computational approach, and experimentally validated the presence of one, sma-miR1867, in S. maritima suggesting that EST information of related species can be used for this purpose. This miRNA was found to be salt-responsive and was downregulated in response to $\mathrm{NaCl}$ application. Although three targets for sma-miR1867 were predicted using ESTs of the halophytes, only one, ubiquitin-protein ligase, could be amplified in S. maritima. A review of the literature revealed that among the three targets, ferredoxin-thioredoxin reductase and CDC6 were responsible for promoting carbon metabolism and cell division, respectively, suggesting that $\mathrm{NaCl}$ promotes these processes in halophytes. Negative regulation of the third target, ubiquitin-protein ligase, by sma-miR1867, nevertheless, indicated that ubiquitin-mediated changes in the abundance of membrane and cytosolic proteins could be important biochemical processes for salt tolerance in halophytes. Downregulation of sma-miR1867 in S. maritima as a response to the use of $\mathrm{NaCl}$ in this study indicates that this miRNA might be important to enable the plant to survive and grow in saline environments.

\section{Conflicts of interest}

The authors declare no conflict of interest.

\section{ACKNOWLEDGMENTS}

The authors are thankful to the Director, Institute of Life Sciences for providing laboratory facilities and financial assistance for the study. S.A. Gharat is thankful to the Dept. of Biotechnology, New Delhi for fellowship.

\section{REFERENCES}

Ambros V, Bartel B, Bartel DP, Burge CB, et al. (2003). A uniform system for microRNA annotation. RNA 9: 277-279. http://dx.doi.org/10.1261/rna.2183803

Buchanan BB, Schürmann P, Wolosiuk RA and Jacquot JP (2002). The ferredoxin/thioredoxin system: from discovery to molecular structures and beyond. Photosynth. Res. 73: 215-222.http://dx.doi.org/10.1023/A:1020407432008

Budak H and Akpinar A (2011). Dehydration stress-responsive miRNA in Brachypodium distachyon: evident by genome-wide screening of microRNAs expression. OMICS 15: 791-799.http://dx.doi.org/10.1089/omi.2011.0073

De Paola D, Cattonaro F, Pignone D and Sonnante G (2012). The miRNAome of globe artichoke: conserved and novel micro RNAs and target analysis. BMC Genomics 13: 41.http://dx.doi.org/10.1186/1471-2164-13-41

Ding D, Zhang L, Wang H, Liu Z, et al. (2009). Differential expression of miRNAs in response to salt stress in maize roots. Ann. Bot. (Lond.) 103: 29-38.http://dx.doi.org/10.1093/aob/mcn205

Feng J, Wang J, Fan P, Jia W, et al. (2015). High-throughput deep sequencing reveals that microRNAs play important roles in salt tolerance of euhalophyte Salicornia europaea. BMC Plant Biol. 15: 63.http://dx.doi.org/10.1186/s12870-015-0451-3

Jian X, Zhang L, Li G, Zhang L, et al. (2010). Identification of novel stress-regulated microRNAs from Oryza sativa L. Genomics 95: 47-55.http://dx.doi.org/10.1016/j.ygeno.2009.08.017

Jones-Rhoades MW and Bartel DP (2004). Computational identification of plant microRNAs and their targets, including a stress-induced miRNA. Mol. Cell 14: 787-799.http://dx.doi.org/10.1016/j.molcel.2004.05.027

Kantar M, Lucas SJ and Budak H (2011). miRNA expression patterns of Triticum dicoccoides in response to shock drought stress. Planta 233: 471-484.http://dx.doi.org/10.1007/s00425-010-1309-4

Khraiwesh B, Pugalenthi G and Fedoroff NV (2013). Identification and analysis of red sea mangrove (Avicennia marina) microRNAs by high-throughput sequencing and their association with stress responses. PLoS One 8: e60774. http://dx.doi.org/10.1371/journal.pone.0060774

Mallory AC and Vaucheret $\mathrm{H}$ (2006). Functions of microRNAs and related small RNAs in plants. Nat. Genet. 38 (Suppl): 
S31-S36.http://dx.doi.org/10.1038/ng1791

Masuda HP, Ramos GBA, de Almeida-Engler J, Cabral LM, et al. (2004). Genome based identification and analysis of the pre-replicative complex of Arabidopsis thaliana. FEBS Lett. 574: 192-202.http://dx.doi.org/10.1016/j.febslet.2004.07.088

Meyers BC, Axtell MJ, Bartel B, Bartel DP, et al. (2008). Criteria for annotation of plant MicroRNAs. Plant Cell 20: 3186-3190. http://dx.doi.org/10.1105/tpc.108.064311

Pandey B, Gupta OP, Pandey DM, Sharma I, et al. (2013). Identification of new stress-induced microRNA and their targets in wheat using computational approach. Plant Signal. Behav. 8: e23932.http://dx.doi.org/10.4161/psb.23932

Peng T, Sun H, Qiao M, Zhao Y, et al. (2014). Differentially expressed microRNA cohorts in seed development may contribute to poor grain filling of inferior spikelets in rice. BMC Plant Biol. 14: 196.http://dx.doi.org/10.1186/s12870-014-0196-4

Stone SL (2014). The role of ubiquitin and the 26S proteasome in plant abiotic stress signaling. Front. Plant Sci. 5: 135. http://dx.doi.org/10.3389/fpls.2014.00135

Sunkar R and Zhu JK (2004). Novel and stress-regulated microRNAs and other small RNAs from Arabidopsis. Plant Cell 16: 2001-2019.http://dx.doi.org/10.1105/tpc.104.022830

Zhang BH, Pan XP, Wang QL, Cobb GP, et al. (2005). Identification and characterization of new plant microRNAs using EST analysis. Cell Res. 15: 336-360.http://dx.doi.org/10.1038/sj.cr.7290302

Zhang BH, Pan XP, Cox SB, Cobb GP, et al. (2006a). Evidence that miRNAs are different from other RNAs. Cell. Mol. Life Sci. 63: 246-254.http://dx.doi.org/10.1007/s00018-005-5467-7

Zhang B, Pan X, Cannon CH, Cobb GP, et al. (2006b). Conservation and divergence of plant microRNA genes. Plant J. 46: 243-259.http://dx.doi.org/10.1111/j.1365-313X.2006.02697.x 